\title{
3 Research Square

\section{Is Age-adjusted Sagittal Parameter Important for Patients Receiving Short Anterior and Oblique Anterolateral Lumbar Surgery? A Single Center Retrospective Review}

\section{Kuan-Kai Tung}

Taichung Veterans General Hospital

Yun-Che Wu

Taichung Veterans General Hospital

Kun-Hui Chen

Taichung Veterans General Hospital

Chien-Chou Pan

Taichung Veterans General Hospital

Wen-Xian Lu

Feng Yuan Hospital Ministry of Health and Welfare

Ning-Chien Chin

Taichung Veterans General Hospital

Cheng-Min Shih

Taichung Veterans General Hospital

\section{Fang-Wei Hsu}

Kuang Tien General Hospital

Cheng-Hung Lee ( $\sim 298 f @ v g h t c . g o v . t w)$

Taichung Veterans General Hospital

\section{Research Article}

Keywords: Adult spinal deformity, Age-adjusted sagittal parameters, ALIF, OLIF, health-related quality of life

Posted Date: August 26th, 2021

DOl: https://doi.org/10.21203/rs.3.rs-840455/v1

License: (c) (1) This work is licensed under a Creative Commons Attribution 4.0 International License. Read Full License 


\section{Abstract}

\section{Background}

Recent studies have suggested the sagittal profiles: SVA, PT, and PI-LL may be age dependent. However, the clinical applications of the age-adjusted parameters remain inconclusive. This study aims to investigate whether age-adjusted sagittal parameter is important for patients receiving short anterolateral lumbar surgery since the recently increased adoption of this surgery.

\section{Methods}

Patients receiving ALIF and OLIF were prospectively collected. The severity of symptoms was measured via the Health-related Quality of Life (HRQOL) score. After the operation, patients were divided into either the "Sufficient group" or "Under group" in accordance with whether they achieved the three age-adjusted parameters. Postoperative clinical outcomes were measured and compared to the pre-OP baselines between the groups at one-year follow-up. Additionally, patients were stratified through the severity cut-off value of each HRQOL score prior to surgery for subgroup analysis.

\section{Results}

All age-adjusted parameters and HRQOLs showed significant improvement after receiving ALIF or OLIF. The sufficient correction rate was 78\%,74\%, and 70\% in age-adjusted SVA, PT, and PI-LL, respectively. EQ5D and ODI demonstrated significant improvements at one-year follow-up upon all patients. Sufficient correction of age-adjusted SVA, PT, and PI-LL revealed better performance in EQ-5D, ODI, and VAS of pain amongst patients with worse pre-OP disability. However, no difference in HRQOLs was observed despite SVA, PT, and PI-LL being sufficiently corrected among the patients with moderate disability. ALIF offered better improvement in EQ-5D compared to OLIF.

\section{Conclusions}

Both ALIF and OLIF can achieve satisfactory clinical results when targeting age-adjusted thresholds. Patients with a worse disability status prior to surgery require sufficient rigorous correction in accordance with age-adjusted targets to achieve better HRQOL.

\section{Introduction}

Spinal malalignment may accompany symptomatic complications, ranging from low back pain to functional disability [1-5]. Mismatch of spinal parameters has been reported as being related to a worse Health-related Quality of Life (HRQOL) $[1-3,6,7]$. Patients who require operative treatment for ASD have suffered from worse spinal-pelvic parameters and clinical symptoms $[3,8,9]$. However, their HRQOL can be significantly improved after surgical correction. 
Disability in patients with ASD has been strongly related to deviation in sagittal alignment [8, 9]. Spinalpelvic alignment varies inconsistently with age and an increased compensation level over time [10]. Ageadjusted sagittal alignment thresholds for the Sagittal Vertical Axis (SVA), Pelvic Tilt (PT) and Pelvic Incidence minus Lumbar Lordosis (PI-LL) had been proposed based upon HRQOL in a multicenter review [11]. Positively exceeding thresholds for spinal profiles were correlated with moderate to severe disability. The concept of an optimal correction goal involving the consideration of age in preoperative (pre-OP) planning has been recently proposed. Scheer et al. reported that under-corrected age-adjusted SVA offered a worse clinical outcome, with no significant difference being observed between matched and overcorrected SVA, as well as an increased potential risk for proximal junctional kyphosis (PJK). Regarding both PT and PI-LL, there were no significant differences in HRQOL amongst any correction achievement $[12,13]$. A Danish study found significant change in Oswestry Disability Index (ODI) after matching correction in the age-adjusted threshold for PI-LL and SVA groups [14].

Studies have shown a correlation between surgical failure and the severity of pre-OP malalignment. Under-corrected patients require greater levels of lower body compensation to maintain postoperative (post-OP) spinal balance [15]. However, those compensations may occur due to lack of a detailed pre-OP assessment surrounding the patient's disability condition and sagittal profiles. Therefore, we assume that patients with severe disability may require rigorous correction goals as compared to patients with moderate disability.

There have been however, several treatment modalities for decompression and correction which have been introduced, including the increasing adoption of the interbody fusion technique which is implemented after failure of conservative strategies [16-18]. Anterolateral lumbar fusion, including anterior lumbar interbody fusion (ALIF), direct lateral lumbar interbody fusion, and oblique lumbar interbody fusion (OLIF) have all been proven to be effective in restoring lumbar lordosis, index disc height, the central canal area, and foraminal area by inserting lordotic angular grafts during a minimally invasive assessment $[18,19]$. Moreover, the advantages which they offer over direct decompression procedures have been established. The benefit of decreased blood loss and the sparing of posterior musculature destruction during surgery, along with post-OP pain being reduced, as well as shorter hospital stays for a faster return to daily activities have all resulted in greater patient satisfaction.

In this study, we aimed to investigate whether age-adjusted sagittal parameter is important for HRQOLs in patients receiving short anterolateral lumbar surgery since the recently increased adoption of this surgery after a full one-year follow-up.

\section{Methods}

\section{Study population}

Patients receiving anterolateral lumbar short fusion, including ALIF and OLIF, for symptomatic lumbar disorders from 2018 to 2021 were identified as the study population from a tertiary medical center. The 
selection criteria for the patients were: (1) fusion levels less than 5 levels; (2) presence of symptomatic low back pain or sciatica which were unresponsive to conservative treatment for more than 6 months (3) spinal deformity defined as a Cobb angle $>20^{\circ}, \mathrm{PT}>25^{\circ}$, or SVA $>50 \mathrm{~mm}$ (4) the pre-OP and post-OP clinical imaging data and follow-up records being complete (5) complete HRQOL assessment at one-year follow-up; (6) pre-OP HRQOL status should be met, including a Visual Analog Scale of Pain in Total (VASP-Total) $>4$, The European Quality of life in 5-dimensional scale (EQ-5D) $>9$, and ODI $>20 \%$. Exclusion criteria including: (1) Loss of follow-up; (2) Spinal deformity due to the presence of malignancy, and/or neuromuscular disease etiology; (3) Patients without full-length lateral spine radiographs at pre-OP and post-OP.

\section{Radiographic Assessment}

Full-length lateral spine radiographs for the kyphosis series (36 inch) at pre-OP visits and the post-OP full one-year follow-up period were analyzed by two well-trained doctors using validated Surgimap surgical planning software (Nemaris Inc., New York, NY, United States) [20]. All radiographic measurements were performed while positioned at a central location based upon standardized techniques, including the, Lumbar Lordosis (LL, lordotic angle from the superior endplate of L1 to the superior endplate of S1), Pelvic Incidence (PI), PI-LL, Pelvic Tilt (PT), Sacral Slope (SS), SVA, and Thoracic Kyphosis (TK) [5].

\section{Outcome measurements}

Standardized self-reported HRQOLs consisted of: (1) EQ-5D; (2) VASP measured in total (VASP-Total), in Back (VASP-Back); and (3) ODI were obtained at pre-OP baseline and one-year follow-up.

\section{Subgroup analysis: the possible effect of pre-OP HRQOL severity}

In the subgroup analysis, patients were stratified by the severity cut-off value of each HRQOL score prior to surgery, with EQ-5D $\geq 12, \mathrm{VASP} \geq 8$, and $\mathrm{ODI} \geq 60$ considered as worse disabilities and will be stratify into the severe symptom groups [21-24].

\section{Surgical techniques}

\section{ALIF procedure}

The ALIF was performed with the patient in the supine position. A longitudinal incision was performed for appropriate spine level exposure. A blunt dissection for retroperitoneal exposure and securing of the inferior epigastric, left common iliac vessels, and genitofemoral nerves was performed. The iliac vessels 
were then exposed and retracted laterally to reveal the fixation level. After $x$-ray positioning, the target annulus fibrosus was resected and intervertebral disc tissue scraped. Curettage and serial distractors were used for efficient disc removal and disc height evaluation. A bone graft was prepared with an allograft or Actifuse. An Implant cage (TM-400 or the Depuy Synthes Syncage system) was inserted under sufficient exposure. Position of the implant was confirmed through x-ray fluoroscopy. The wound was then closed, and adequate hemostasis was performed.

\section{OLIF procedure}

The OLIF was performed with the patient positioned in the right-lateral position (Figure 1). A longitudinal incision on the front line of the iliac crest was made for appropriate spine level exposure before the psoas muscle was gently retracted posteriorly. After x-ray positioning, the target annulus fibrosus was resected and intervertebral disc tissue scraped. Serial trial was applied for optimal cage size. Endplate damage was avoided during the procedure. The Implant cage (Medtronic CLYDESDALE spine system) was inserted under sufficient exposure (Figure 2). Position of the implant was confirmed by $\mathrm{x}$-ray fluoroscopy (Figure 3). The wound was then closed, and adequate hemostasis performed.

\section{Patient Age-Adjustment Grouping}

Age-specific sagittal parameters were calculated according to a previously published formula by Lafage et al. (Figure 4) [11]. After the operation, patients were divided into either the "Sufficient group" or "Under group" in accordance with the achievement or under treatment of the three age-adjusted parameters: SVA, PT, and PI-LL (Figure 5). Post-OP clinical outcomes were measured and compared to the pre-OP baseline between groups at one-year follow-up.

\section{Statistical Analysis}

Demographics factors and clinical data, including age, body mass index (BMI), gender, operation technique, and length of postoperative hospital stay were recorded. Normality of data was determined via the Shapiro-Wilk test. Continuous variables were described with the mean and standard deviation. Intragroup HRQOL means analyses were conducted via one-way analysis of variance (ANOVA), Kruskall-Wallis test, or Mann-Whitney u test according to appropriate models. Statistical analyses were two-sided and P $<0.05$ was considered statistically significant. All statistical analyses were performed using SPSS version 24 (IBM, Armonk, New York, USA).

\section{Results}

\section{Patient Population Demographics}


We enrolled 50 patients at 72 levels with patients receiving anterolateral LIFs for symptomatic lumbar disorders in the baseline and post-OP analysis (Table 1). The mean age of the patients was $59.5 \pm 13$ years. Amongst the patients, $29(58 \%)$ patients were female and $30(60 \%)$ of them received ALIF. The mean BMI was $25.9 \pm 3 \mathrm{~kg} / \mathrm{m}^{2}$, and post-OP hospital stay was $7.9 \pm 2.9$ days. Most of the patients were diagnosed with spondylolisthesis (64\%) and spondylosis (34\%). Most of the patients received single-level LIF (70\%), targeting L5-S1 in ALIF and L4-5 in OLIF, respectively. All patients were successfully discharged after the hospital course and followed up for a full one year after the operation. No patient received revision spinal surgery during one-year follow-up. There was no spinal nerve, major vessel, peritoneal, or urinary injury experienced after the operation.

\section{Spinal-pelvic Parameters Analysis and HRQOL Analysis amongst All Patients}

The comparison of radiographic sagittal parameters and HRQOL between pre-OP and one-year were made (Table 2). Sagittal parameters including LL, PI-LL, and SVA reached statistical improvement. All HRQOLs were statistically improved. The mean of SVA decreased from $60.3 \pm 41.9$ (pre-OP) to $32.5 \pm 35$ (one-year) $\mathrm{mm}$; PT decreased from $18.8^{\circ} \pm 9.2^{\circ}$ to $18.3^{\circ} \pm 13.2^{\circ}$; and PI-LL decreased from $12.5^{\circ} \pm 17.6^{\circ}$ to $8.3^{\circ} \pm 14.8^{\circ}$. The EQ-5D score decreased from $11.6 \pm 1.1$ to $7.3 \pm 2.3$; ODI score decreased from $53.9 \pm 12.7 \%$ to $26.5 \pm 16.9 \%$; VASP-Total decreased from $8.2 \pm 1.4$ to $2.5 \pm 1.9$; and the VASP-Back decreased from $7.7 \pm 2.5$ to $2.6 \pm 2.1$ ( $p<0.01$ for all).

\section{Comparison of outcomes between the ALIF and OLIF}

The baseline demographics and surgical outcomes were compared between ALIF and OLIF at one-year follow up and are displayed in Table 3. The ALIF group experienced better outcomes in EQ-5D (ALIF vs OLIF: $6.8 \pm 2.2$ vs $8.1 \pm 2.2, p=0.04$ ) at one-year follow-up. No statistical significance was reached in sagittal alignment parameters, ODI, VASP-Total, or VASP-Back.

\section{Outcomes after achieving of age-adjusted thresholds}

Patients achieving age-adjusted thresholds were 39 (78\%), 37 (74\%), and 35 (70\%) in SVA, PT, and PI-LL amongst the 50 patients, respectively (Table 4). Statistical differences were found in EQ-5D after sufficiently corrected SVA and PT by targeting their age-adjusted threshold. The ODI was statistically different only by achieving the age-adjusted SVA threshold.

In the subgroup analysis considering the effect of severity of the symptoms prior to the surgery, there were $76 \%, 68 \%, 74 \%$, and $78 \%$ patients suffering from worse disability of EQ-5D, ODI, VASP-Total and VASP-Back prior to the operation. Amongst patients with a worse disability, sufficient correction of ageadjusted SVA, PT, and PI-LL showed statistically better outcomes in EQ-5D, ODI, VASP-Total and VASP- 
Back as compared to the Under group (Table 5). Although the EQ-5D and VASP-Total was better in PI-LL and PT group after sufficient correction, no statistical significance was reached. Contrarily, sufficient correction of age-adjusted SVA, PT, and PI-LL in the moderately disabled group presented a general improvement trend of HRQOLs without reaching statistically significant differences (Table 6).

\section{Discussion}

In the current study, we found improvements of EQ-5D and ODI after sufficient correction targeting ageadjusted SVA and PT amongst patients receiving short anterolateral LIFs for symptomatic lumbar disorders at one-year follow-up. Moreover, subgroup analysis by stratifying patients with pre-OP HRQOL status demonstrated a worse pre-OP HRQOL score could have their condition significantly improved whilst being sufficiently corrected in accordance with age-adjusted thresholds of SVA, PT, and PI-LL. We have also proved the efficacy of short anterolateral lumbar fusion with posterior instrumentation, including the ALIF and OLIF techniques, by improving the HRQOL for those in the study cohort at full oneyear follow up. The ALIF group showed better EQ-5D at one-year follow up than the OLIF group. According to our knowledge, this is the first study reporting the surgical outcomes of short anterolateral LIFs targeting age-adjusted thresholds.

Previous studies had demonstrated the clinical efficacy of anterior lumbar fusion. Patients receiving ALIF experienced an $86 \%$ successful clinical outcome and an overall $94 \%$ fusion rate after two-year follow up [25]. Despite the great heterogeneity between the radiographic outcomes which resulted from the studies, the consensus that ALIF surgery offers satisfactory functional improvements in disability, pain, and daily health has been established for years [26]. As for OLIF, significant improvement in HRQOL with marked advantages such as shorter surgery times, decreased intraoperative blood loss, and improved post-OP pain relief when compared to traditional posterior lumbar fusion techniques have been reported. These merits are in accordance with our surgical outcomes. Expectedly, both sagittal parameters and HRQOL offered significant improvement at one-year after surgery in the current study (Table 2), implying that both ALIF and OLIF successfully mitigated the clinical symptoms. By comparing ALIF to OLIF, no radiographic or HRQOLs results were significantly different, except for EQ-5D in the ALIF group (Table 3).

Sagittal radiographic parameters such as SVA, PT, and PI-LL mismatch are considered the key factors which impact disability and are modified in accordance with age [11]. However, surgical principles regarding these thresholds remain controversial for their clinical application. Scheer et al. reported that under-corrected age-adjusted SVA offered a worse clinical outcome, with no significant difference being observed between matched and overcorrected SVA, as well as an increased potential risk for proximal junctional kyphosis (PJK). Regarding both PT and PI-LL, there were no significant differences in HRQOL amongst any correction achievement $[12,13]$. A Danish study found significant change in ODI after matching correction in the age-adjusted threshold for PI-LL and SVA groups [14]. They suggested that the age appropriate SVA target prior to surgery will be the most crucial implication for future practice. In our study, we found a similar trend of improvement of EQ-5D, ODI, VASP in total and back by stratifying them in accordance with the achievement of age-adjusted thresholds, including SVA and PT. The preliminary 
result for HRQOL outcomes targeting age-adjusted threshold in the current study (Table 4) is in line with previous conclusions. However, this result was difficult to apply in the clinical practice since SVA was difficult to be managed and monitored intraoperatively. Some studies challenged the overemphasize of the SVA as being the most important quality-of-life parameter. They consider that SVA should only be used to compare the balance situation of a patient over time, or before and after surgical treatment, not between a series of patients due to the diversity of their pelvic incidence angles [5]. Additionally, not until the compensating mechanisms of PT and PI-LL have been exhausted that the SVA will become clinically imbalanced [14]. Intervention timing prior to complete compensation of SVA may provide more opportunity for rescuing the spinal alignment $[27,28]$. Hence, the role of age-adjusted PT and PI-LL requires further evaluation regarding their involvement in this compensation mechanism and ideal target.

On the other hand, we believe patients with different baseline symptoms may require alternative correction principles. By stratifying our patients in accordance with the pre-OP severity of symptoms, patients with a worse disability before surgery experienced better HRQOLs once they were sufficiently corrected through targeting of the age-adjusted threshold (Table 5). However, it may remain unnecessary for patients with moderate symptoms to target the age-ideal threshold since no significance was reached between the correction group (Table 6). In addition, PI-LL can be monitored and manipulated intraoperatively, since $\mathrm{PI}$ is commonly used to define spinopelvic morphotypes due to its constancy. By adjusting LL during intraoperative imaging, we will be able to correct PI-LL mismatch during operation. The correlation of PI-LL mismatch measurement between the intraoperative lumbar X-ray and post-OP standing full spine X-ray has been assessed and adjusted, respectively. [28] We consider the consistently baseline sagittal malalignment may be related to a worse baseline symptomatic condition. Patientspecific thresholds mitigate failure risk by individualizing the surgical goal. Moreover, compensatory recruitment such as posterior pelvic shift, knee flexion, and thoracic hypokyphosis were unable to regain age-ideal curvature after under-correction of SVA, PT, and PI-LL mismatch [15]. The loss of flexibility and consistently larger baseline sagittal malalignment explains the reason for under-correction.

The limitations of the study are worth discussing. Firstly, the small sample size and short follow-up for only one-year may inevitably affect the outcomes. However, the result of this study has proven the efficacy of short segments anterolateral LIFs in sagittal alignment and HRQOLs. Secondly, covariate analysis was unable to be controlled since baseline comorbidities were not included in our analysis. Finally, short segments LIFs may provide insufficient contribution to the sagittal alignment. Moreover, the surgical indication for L5-S1 LIF was confined only to the ALIF procedures. Nevertheless, we have provided preliminary ideas for intraoperative age-adjusted alignment targets for anterolateral lumbar fusion, and a further monitoring method will be conducted in a prospective design setting.

\section{Conclusion}

Short anterolateral LIFs, including ALIF and OLIF, can achieve satisfactory clinical results targeting ageadjusted thresholds for symptomatic lumbar disorders patients, including SVA, PT, and PI-LL. Amongst those patients with a worse disability prior to surgery, a rigorous sufficient correction may be required in 
accordance with age-adjusted targets to achieve better HRQOL. With due attention paid to intra-OP manipulation for age-adjusted targets, adequate methods should continue to be studied in the future.

\section{Abbreviations}

ALIF: Anterior Lateral Interbody Fusion; BMI: body mass index; EQ-5D: European Quality of life in 5dimensional scale ; HRQOL: Health-related Quality of Life; LIFs: Lumbar Interbody Fusions; LL: lumbar Iordosis; OLIF: Oblique Lateral Interbody Fusion; OP: Operative; ODI: Oswestry Disability Index; PI: Pelvic Incidence; PI-LL: Pelvic Incidence minus Lumbar Lordosis; PT: Pelvic Tilt; PJK: proximal junctional kyphosis; SVA: Sagittal Vertical Axis; SS: Sacral Slope; VASP: Visual Analog Scale of Pain; TK: Thoracic Kyphosis; TLIF: Transforaminal Lateral Interbody Fusion;

\section{Declarations}

\section{Ethics approval and consent to participate}

This current study has been approved by the Research Ethics Committee of Taichung Veterans General Hospital in Taiwan (TCVGH-IRB-CE20218B), with all patients signing the written informed consent. All methods were performed in accordance with the relevant guidelines and regulations approved by the Research Ethics Committee of Taichung Veterans General Hospital.

\section{Consent for publication}

Not Applicable

\section{Availability of data and materials}

The original contributions presented in the study are included in the article. Further inquiries can be directed to the corresponding author.

\section{Competing interests}

The authors have no relevant financial or non-financial interests to disclose.

\section{Funding}

The authors did not receive support from any organization for the submitted work.

\section{Author contributions}


$\mathrm{K}-\mathrm{KT}$, and $\mathrm{Y}-\mathrm{CW}$ had full access to all the data in the study and take responsibility for both the integrity of the data and the accuracy of the data analysis. K-KT, Y-CW, and C-HL: study concept and design, acquisition of data. K-KT: statistical analysis. KKT, Y-CW: drafting of the manuscript. K-HC, C-CP, W-XL, N$\mathrm{CC}, \mathrm{C}-\mathrm{MS}$, F-WH, and C-HL: study supervision. All authors: interpretation of data; critical revision of the manuscript for important intellectual content.

\section{Acknowledgements}

We would like to give special thanks to the language editorial support provided by Taichung Veterans General Hospital.

\section{Patient and public involvement}

All data were extracted from Taichung Veterans General Hospital. Neither the patients nor the public were involved.

\section{References}

1. Schwab F, Patel A, Ungar B, Farcy JP, Lafage V: Adult spinal deformity-postoperative standing imbalance: how much can you tolerate? An overview of key parameters in assessing alignment and planning corrective surgery. Spine (Phila Pa 1976) 2010, 35(25):2224-2231.

2. Bess $S$, Protopsaltis TS, Lafage V, Lafage R, Ames CP, Errico T, Smith JS: Clinical and Radiographic Evaluation of Adult Spinal Deformity. Clin Spine Surg 2016, 29(1):6-16.

3. Glassman SD, Bridwell K, Dimar JR, Horton W, Berven S, Schwab F: The impact of positive sagittal balance in adult spinal deformity. Spine (Phila Pa 1976) 2005, 30(18):2024-2029.

4. Schwab F, Lafage V, Patel A, Farcy JP: Sagittal plane considerations and the pelvis in the adult patient. Spine (Phila Pa 1976) 2009, 34(17):1828-1833.

5. Le Huec JC, Thompson W, Mohsinaly Y, Barrey C, Faundez A: Sagittal balance of the spine. European Spine Journal 2019, 28(9):1889-1905.

6. Schwab FJ, Smith VA, Biserni M, Gamez L, Farcy JP, Pagala M: Adult scoliosis: a quantitative radiographic and clinical analysis. Spine (Phila Pa 1976) 2002, 27(4):387-392.

7. Choi HY, Hyun SJ, Kim KJ, Jahng TA, Kim HJ: Radiographic and Clinical Outcomes Following Pedicle Subtraction Osteotomy: Minimum 2-Year Follow-Up Data. J Korean Neurosurg Soc 2020, 63(1):99107.

8. Jang JS, Lee SH, Min JH, Kim SK, Han KM, Maeng DH: Surgical treatment of failed back surgery syndrome due to sagittal imbalance. Spine (Phila Pa 1976) 2007, 32(26):3081-3087. 
9. Schwab FJ, Blondel B, Bess S, Hostin R, Shaffrey Cl, Smith JS, Boachie-Adjei O, Burton DC, Akbarnia BA, Mundis GM et al: Radiographical spinopelvic parameters and disability in the setting of adult spinal deformity: a prospective multicenter analysis. Spine (Phila Pa 1976) 2013, 38(13):E803-812.

10. Passias PG, Horn SR, Frangella NJ, Poorman GW, Vasquez-Montes D, Diebo BG, Bortz CA, Segreto FA, Moon JY, Zhou PL et al: Full-Body Analysis of Adult Spinal Deformity Patients' Age-Adjusted Alignment at 1 Year. World Neurosurg 2018, 114:e775-e784.

11. Lafage R, Schwab F, Challier V, Henry JK, Gum J, Smith J, Hostin R, Shaffrey C, Kim HJ, Ames C et al: Defining Spino-Pelvic Alignment Thresholds: Should Operative Goals in Adult Spinal Deformity Surgery Account for Age? Spine (Phila Pa 1976) 2016, 41(1):62-68.

12. Scheer JK, Lafage R, Schwab FJ, Liabaud B, Smith JS, Mundis GM, Hostin R, Shaffrey Cl, Burton DC, Hart RA et al: Under Correction of Sagittal Deformities Based on Age-adjusted Alignment Thresholds Leads to Worse Health-related Quality of Life Whereas Over Correction Provides No Additional Benefit. Spine (Phila Pa 1976) 2018, 43(6):388-393.

13. Lafage R, Schwab F, Glassman S, Bess S, Harris B, Sheer J, Hart R, Line B, Henry J, Burton D et al: Age-Adjusted Alignment Goals Have the Potential to Reduce PJK. Spine (Phila Pa 1976) 2017, 42(17):1275-1282.

14. Hallager DW, Hansen LV, Dragsted CR, Peytz N, Gehrchen M, Dahl B: A Comprehensive Analysis of the SRS-Schwab Adult Spinal Deformity Classification and Confounding Variables: A Prospective, NonUS Cross-sectional Study in 292 Patients. Spine (Phila Pa 1976) 2016, 41(10):E589-597.

15. Passias PG, Jalai CM, Diebo BG, Cruz DL, Poorman GW, Buckland AJ, Day LM, Horn SR, Liabaud B, Lafage R et al: Full-Body Radiographic Analysis of Postoperative Deviations From Age-Adjusted Alignment Goals in Adult Spinal Deformity Correction and Related Compensatory Recruitment. Int J Spine Surg 2019, 13(2):205-214.

16. Mobbs RJ, Phan K, Malham G, Seex K, Rao PJ: Lumbar interbody fusion: techniques, indications and comparison of interbody fusion options including PLIF, TLIF, MI-TLIF, OLIF/ATP, LLIF and ALIF. $J$ Spine Surg 2015, 1(1):2-18.

17. Allain J, Dufour T: Anterior lumbar fusion techniques: ALIF, OLIF, DLIF, LLIF, IXLIF. Orthop Traumatol Surg Res 2020, 106(1s):S149-s157.

18. Watkins RGt, Hanna R, Chang D, Watkins RG, 3rd: Sagittal alignment after lumbar interbody fusion: comparing anterior, lateral, and transforaminal approaches. J Spinal Disord Tech 2014, 27(5):253256.

19. Anand N, Alayan A, Agrawal A, Kahwaty S, Nomoto E, Khandehroo B: Analysis of Spino-Pelvic Parameters and Segmental Lordosis with L5-S1 Oblique Lateral Interbody Fusion at the Bottom of a Long Construct in Circumferential Minimally Invasive Surgical Correction of Adult Spinal Deformity. World Neurosurg 2019, 130:e1077-e1083.

20. Akbar M, Terran J, Ames CP, Lafage V, Schwab F: Use of Surgimap Spine in sagittal plane analysis, osteotomy planning, and correction calculation. Neurosurg Clin N Am 2013, 24(2):163-172. 
21. Woo A, Lechner B, Fu T, Wong CS, Chiu N, Lam H, Pulenzas N, Soliman H, DeAngelis C, Chow E: Cut points for mild, moderate, and severe pain among cancer and non-cancer patients: a literature review. Ann Palliat Med 2015, 4(4):176-183.

22. Tonosu J, Takeshita K, Hara N, Matsudaira K, Kato S, Masuda K, Chikuda H: The normative score and the cut-off value of the Oswestry Disability Index (ODI). Eur Spine J 2012, 21(8):1596-1602.

23. Herdman M, Gudex C, Lloyd A, Janssen M, Kind P, Parkin D, Bonsel G, Badia X: Development and preliminary testing of the new five-level version of EQ-5D (EQ-5D-5L). Qual Life Res 2011, 20(10):1727-1736.

24. Devlin NJ, Brooks R: EQ-5D and the EuroQol Group: Past, Present and Future. Appl Health Econ Health Policy 2017, 15(2):127-137.

25. Rao PJ, Loganathan A, Yeung V, Mobbs RJ: Outcomes of anterior lumbar interbody fusion surgery based on indication: a prospective study. Neurosurgery 2015, 76(1):7-23; discussion 23-24.

26. Giang G, Mobbs R, Phan S, Tran TM, Phan K: Evaluating Outcomes of Stand-Alone Anterior Lumbar Interbody Fusion: A Systematic Review. World Neurosurg 2017, 104:259-271.

27. Diebo BG, Henry J, Lafage V, Berjano P: Sagittal deformities of the spine: factors influencing the outcomes and complications. Eur Spine J 2015, 24 Suppl 1:S3-15.

28. Praeger AJ, Wang YYI, Goldschlager T: Intraoperatively predicting postoperative sagittal balance using intraoperative X-rays. J Clin Neurosci 2019, 63:48-54.

\section{Tables}


Table 1

Demographic data of the study population stratified by lumbar interbody fusion types

\begin{tabular}{|c|c|c|c|c|}
\hline & ALIF & OLIF & Overall & $P$ value \\
\hline Patient number & 30 & 20 & 50 & \\
\hline Age & $56.5 \pm 15$ & $64.2 \pm 7.2$ & $59.5 \pm 13$ & 0.11 \\
\hline BMI & $25.5 \pm 2.7$ & $26.6 \pm 3.4$ & $25.9 \pm 3$ & 0.26 \\
\hline \multicolumn{5}{|l|}{ Gender } \\
\hline Female & $18(60 \%)$ & $11(55 \%)$ & $29(58 \%)$ & \multirow[t]{2}{*}{0.66} \\
\hline Male & $12(40 \%)$ & $9(45 \%)$ & $21(42 \%)$ & \\
\hline Hospital stays & $8 \pm 3.1$ & $7.9 \pm 2.6$ & $7.9 \pm 2.9$ & 0.65 \\
\hline \multicolumn{5}{|l|}{ Pre-OP diagnosis } \\
\hline Spondylolisthesis & $18(60 \%)$ & $14(70 \%)$ & $32(64 \%)$ & \multirow[t]{3}{*}{0.74} \\
\hline Spondylosis & $12(40 \%)$ & $5(25 \%)$ & $17(34 \%)$ & \\
\hline Infection & $0(0 \%)$ & $1(5 \%)$ & $1(2 \%)$ & \\
\hline \multicolumn{5}{|l|}{ Index fusion level } \\
\hline L2-L3 & $1(2.2 \%)$ & $2(7.4 \%)$ & $3(41.2 \%)$ & \multirow[t]{4}{*}{0.08} \\
\hline L3-L4 & $6(13.3 \%)$ & $11(40.7 \%)$ & $17(23.6 \%)$ & \\
\hline L4-L5 & $15(33.3 \%)$ & $14(51.9 \%)$ & $29(40.3 \%)$ & \\
\hline L5-S1 & $23(51.1 \%)$ & $0(0 \%)$ & $23(31.9 \%)$ & \\
\hline Total fusion levels & 45 & 27 & 72 & \\
\hline \multicolumn{5}{|c|}{ Fusion level (per person) } \\
\hline 1 & $20(66.7 \%)$ & $15(75 \%)$ & $35(70 \%)$ & \multirow[t]{4}{*}{0.41} \\
\hline 2 & $6(20 \%)$ & $3(15 \%)$ & $9(18 \%)$ & \\
\hline 3 & $3(10 \%)$ & $2(10 \%)$ & $6(12 \%)$ & \\
\hline 4 & $1(3.3 \%)$ & 0 & 0 & \\
\hline
\end{tabular}


Table 2

Comparison of sagittal alignments and HRQOLs between one-year follow-up and pre-OP baseline

\begin{tabular}{|c|c|c|c|c|}
\hline Parameters & Pre-OP & $1 Y$ & Pair-T value & $P$ value \\
\hline LL & $41.2 \pm 18.9$ & $45.3 \pm 17.4$ & 1.9 & $0.04^{*}$ \\
\hline $\mathrm{PI}$ & $52.2 \pm 13.2$ & $52.2 \pm 13.8$ & 0.1 & 0.96 \\
\hline PI-LL & $12.5 \pm 17.6$ & $8.3 \pm 14.8$ & -2.0 & $0.03^{\star}$ \\
\hline PT & $18.8 \pm 9.2$ & $18.3 \pm 13.2$ & -0.4 & 0.68 \\
\hline SS & $33.3 \pm 10.5$ & $33.9 \pm 13.6$ & 0.4 & 0.69 \\
\hline SVA & $60.3 \pm 41.9$ & $32.5 \pm 35$ & -3.9 & $<0.01 \star \star$ \\
\hline TK & $18.8 \pm 12.6$ & $21.2 \pm 9.8$ & 1.7 & 0.09 \\
\hline EQ-5D & $11.6 \pm 1.1$ & $7.3 \pm 2.3$ & -14.0 & $<0.01 * \star$ \\
\hline ODI (\%) & $53.9 \pm 12.7$ & $26.5 \pm 16.9$ & -12.6 & $<0.01^{\star \star}$ \\
\hline VASP-Total & $8.2 \pm 1.4$ & $2.5 \pm 1.9$ & -13.5 & $<0.01^{\star \star}$ \\
\hline VASP-Back & $7.7 \pm 2.5$ & $2.6 \pm 2.1$ & -19.1 & $<0.01^{\star \star}$ \\
\hline \multicolumn{5}{|c|}{$\begin{array}{l}\text { Continuous data are expressed as mean } \pm \text { SD. Categorical data were expressed number and } \\
\text { percentage. *p< } 0.05,{ }^{\star *} \mathrm{p}<0.01 \text { : consider statistically significant for Paired Sample t-test. LL: Lumbar } \\
\text { Lordosis; PI: Pelvic Incidence; PI-LL: Pelvic Incidence minus Lumbar Lordosis; PT: Pelvic Tilt; SS: } \\
\text { Sacral Slope; SVA: Sagittal Vertical Axis; TK: Thoracic Kyphosis; EQ-5D: European Quality of life in 5- } \\
\text { dimensional scale; ODI: Oswestry Disability Index; VASP-Total: Visual Analog Scale of Pain in Total; } \\
\text { VASP-Back: Visual Analog Scale of Pain in back. }\end{array}$} \\
\hline
\end{tabular}


Table 3

Comparison of sagittal alignments and HRQOLs between ALIF and OLIF at one-year follow-up

\begin{tabular}{|c|c|c|c|}
\hline Parameters & ALIF & OLIF & $P$ value \\
\hline$N$ & 30 & 20 & \\
\hline LL & $44.6 \pm 19.5$ & $41.5 \pm 13.8$ & 0.39 \\
\hline $\mathrm{PI}$ & $53.5 \pm 13.2$ & $50.2 \pm 13.3$ & 0.39 \\
\hline PI-LL & $9 \pm 15.9$ & $7.2 \pm 13.4$ & 0.74 \\
\hline PT & $19.6 \pm 9.1$ & $17.6 \pm 9.6$ & 0.36 \\
\hline SS & $33.9 \pm 12.1$ & $32.5 \pm 7.7$ & 0.49 \\
\hline SVA & $31.2 \pm 35.7$ & $34.5 \pm 34.7$ & 0.66 \\
\hline TK & $21.4 \pm 10.7$ & $20.9 \pm 8.6$ & 0.87 \\
\hline EQ-5D & $6.8 \pm 2.2$ & $8.1 \pm 2.2$ & $0.04 *$ \\
\hline ODI (\%) & $23.3 \pm 17.9$ & $31.1 \pm 14.3$ & 0.08 \\
\hline VASP-Total & $2.5 \pm 1.9$ & $2.5 \pm 2$ & 0.99 \\
\hline VASP-Back & $2.6 \pm 2.2$ & $2.5 \pm 2$ & 0.87 \\
\hline \multicolumn{4}{|c|}{$\begin{array}{l}\text { Continuous data are expressed as mean } \pm \text { SD. }{ }^{*} p<0.05,{ }^{*} p<0.01 \text { : consider statistically significant } \\
\text { for comparison of ALIF and OLIF. LL: Lumbar Lordosis; PI: Pelvic Incidence; PI-LL: Pelvic Incidence } \\
\text { minus Lumbar Lordosis; PT: Pelvic Tilt; SS: Sacral Slope; SVA: Sagittal Vertical Axis; TK: Thoracic } \\
\text { Kyphosis; EQ-5D: European Quality of life in 5-dimensional scale; ODI: Oswestry Disability Index; } \\
\text { VASP-Total: Visual Analog Scale of Pain in Total; VASP-Back: Visual Analog Scale of Pain in back. }\end{array}$} \\
\hline
\end{tabular}


Table 4

One-year HRQOLs for all patients targeting age-adjusted thresholds

\begin{tabular}{|c|c|c|c|c|c|}
\hline & $\mathbf{N}$ & EQ-5D & ODI (\%) & VASP-Total & VASP-Back \\
\hline \multicolumn{6}{|l|}{ SVA } \\
\hline Under & 11 & $8.6 \pm 1.5$ & $39 \pm 15.8$ & $3.5 \pm 1.7$ & $4.4 \pm 1.3$ \\
\hline Sufficient & 39 & $6.4 \pm 2.3$ & $22.9 \pm 17.1$ & $1.8 \pm 1.9$ & $1.7 \pm 1.9$ \\
\hline $\mathrm{p}$ & & $0.04^{*}$ & $<0.01 * \star$ & 0.24 & 0.05 \\
\hline \multicolumn{6}{|l|}{ PT } \\
\hline Under & 13 & $8.2 \pm 1.6$ & $37.7 \pm 16.5$ & $3.7 \pm 2.2$ & $3.4 \pm 1.9$ \\
\hline Sufficient & 37 & $6.5 \pm 2.3$ & $22.6 \pm 16.9$ & $2 \pm 2$ & $2 \pm 2.1$ \\
\hline $\mathrm{p}$ & & $0.02^{*}$ & 0.12 & 0.68 & 0.34 \\
\hline \multicolumn{6}{|l|}{ PI-LL } \\
\hline Under & 15 & $7.7 \pm 1.9$ & $33.7 \pm 18.8$ & $3.3 \pm 2.3$ & $3.1 \pm 2.3$ \\
\hline Sufficient & 35 & $6.6 \pm 2.4$ & $24.7 \pm 17.2$ & $2.4 \pm 2.1$ & $2 \pm 1.9$ \\
\hline$p$ & & 0.15 & 0.17 & 0.71 & 0.09 \\
\hline \multicolumn{6}{|c|}{$\begin{array}{l}\text { Continuous data are expressed as mean } \pm \text { SD. }{ }^{*} p<0.05,{ }^{*} \mathrm{p}<0.01 \text { : consider statistically significant. } \\
\text { EQ-5D: European Quality of life in 5-dimensional scale; ODI: Oswestry Disability Index; VASP-Total: } \\
\text { Visual Analog Scale of Pain in Total; VASP-Back: Visual Analog Scale of Pain in back. }\end{array}$} \\
\hline
\end{tabular}


Table 5

One-year HRQOLs for patients being Under-, or Sufficient-corrected targeting age-adjusted thresholds with severe pre-OP disability

\begin{tabular}{|c|c|c|c|c|c|c|c|c|}
\hline & $\mathbf{N}$ & EQ-5D & $\mathbf{N}$ & ODI (\%) & $\mathbf{N}$ & VASP-Total & $\mathbf{N}$ & VASP-Back \\
\hline \multicolumn{9}{|l|}{ SVA } \\
\hline Under & 15 & $8.9 \pm 1.4$ & 12 & $35.6 \pm 13.2$ & 14 & $3.5 \pm 1.6$ & 16 & $4.3 \pm 1.4$ \\
\hline Sufficient & 23 & $6.8 \pm 2.5$ & 22 & $25.8 \pm 15.3$ & 23 & $2 \pm 1.8$ & 23 & $2.1 \pm 1.8$ \\
\hline$p$ & & $0.01 *$ & & $0.04 *$ & & $0.02^{\star}$ & & $<0.01^{\star *}$ \\
\hline \multicolumn{9}{|l|}{ PT } \\
\hline Under & 16 & $8.6 \pm 1.6$ & 13 & $36.6 \pm 12.5$ & 16 & $2.9 \pm 1.6$ & 16 & $4.1 \pm 1.3$ \\
\hline Sufficient & 22 & $7 \pm 2.6$ & 21 & $24.7 \pm 15.1$ & 21 & $2.2 \pm 2.1$ & 23 & $2.3 \pm 2$ \\
\hline$p$ & & $0.04^{\star}$ & & $0.02 *$ & & 0.28 & & $0.01 *$ \\
\hline \multicolumn{9}{|l|}{ PI-LL } \\
\hline Under & 13 & $8.4 \pm 1.9$ & 11 & $39 \pm 13.2$ & 13 & $3.5 \pm 1.6$ & 14 & $4.4 \pm 1.3$ \\
\hline Sufficient & 25 & $7.3 \pm 2.5$ & 23 & $24.5 \pm 13.9$ & 24 & $2 \pm 1.9$ & 25 & $2.2 \pm 1.8$ \\
\hline $\mathrm{p}$ & & 0.19 & & $0.01 *$ & & $0.03^{*}$ & & $<0.01 * \star$ \\
\hline
\end{tabular}


Table 6

One-year HRQOLs for patients being Under-, or Sufficient-corrected targeting age-adjusted thresholds with moderate pre-OP disability

\begin{tabular}{|c|c|c|c|c|c|c|c|c|}
\hline & $\mathbf{N}$ & EQ-5D & $\mathbf{N}$ & ODI (\%) & $\mathbf{N}$ & VASP-Total & $\mathbf{N}$ & VASP-Back \\
\hline \multicolumn{9}{|l|}{ SVA } \\
\hline Under & 2 & $6 \pm 0$ & 5 & $23.1 \pm 12.5$ & 3 & $3.7 \pm 1.2$ & 1 & $1 \pm 0$ \\
\hline Sufficient & 10 & $6.3 \pm 1.8$ & 11 & $13.5 \pm 14.2$ & 10 & $1.8 \pm 2.1$ & 10 & $1.4 \pm 2.1$ \\
\hline$p$ & & 0.65 & & 0.06 & & 0.16 & & 0.73 \\
\hline \multicolumn{9}{|l|}{ PT } \\
\hline Under & 3 & $6.7 \pm 1.2$ & 6 & $20.4 \pm 13$ & 3 & $3.7 \pm 1.2$ & 3 & $0.3 \pm 0.6$ \\
\hline Sufficient & 9 & $6.1 \pm 1.8$ & 10 & $14.2 \pm 14.7$ & 10 & $1.8 \pm 2.1$ & 8 & $1.8 \pm 2.2$ \\
\hline$p$ & & 0.24 & & 0.15 & & 0.16 & & 0.37 \\
\hline \multicolumn{9}{|l|}{ PI-LL } \\
\hline Under & 4 & $6.8 \pm 1.5$ & 6 & $17.4 \pm 15$ & 4 & $2 \pm 2.4$ & 3 & $1.9 \pm 2.1$ \\
\hline Sufficient & 8 & $6 \pm 1.7$ & 10 & $16 \pm 14.2$ & 9 & $2.3 \pm 2$ & 8 & $0 \pm 0$ \\
\hline$p$ & & 0.32 & & 0.74 & & 0.81 & & 0.09 \\
\hline
\end{tabular}

\section{Figures}




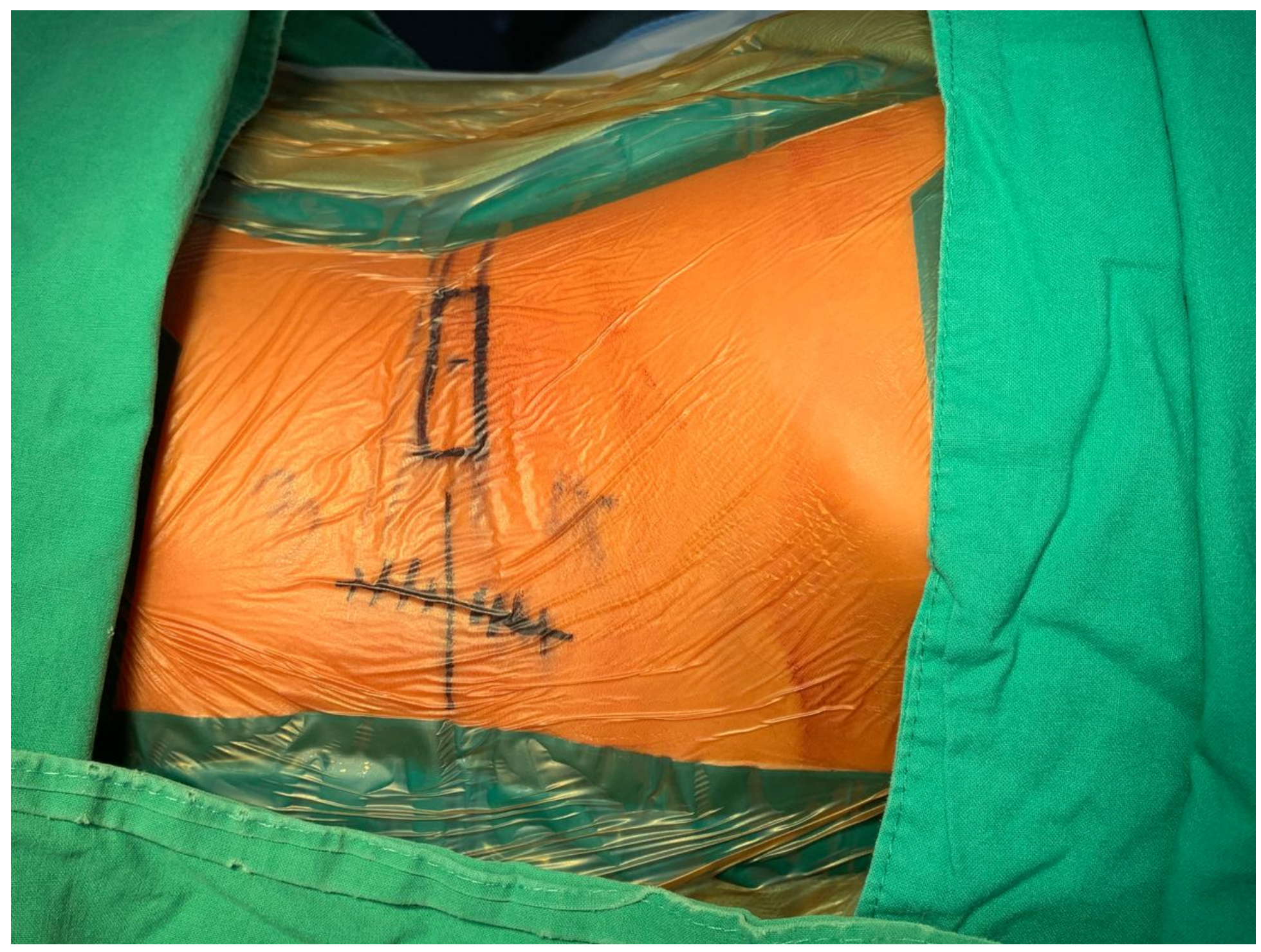

\section{Figure 1}

In the OLIF procedure, patient was positioned in the right-lateral position before the incision marker was made 


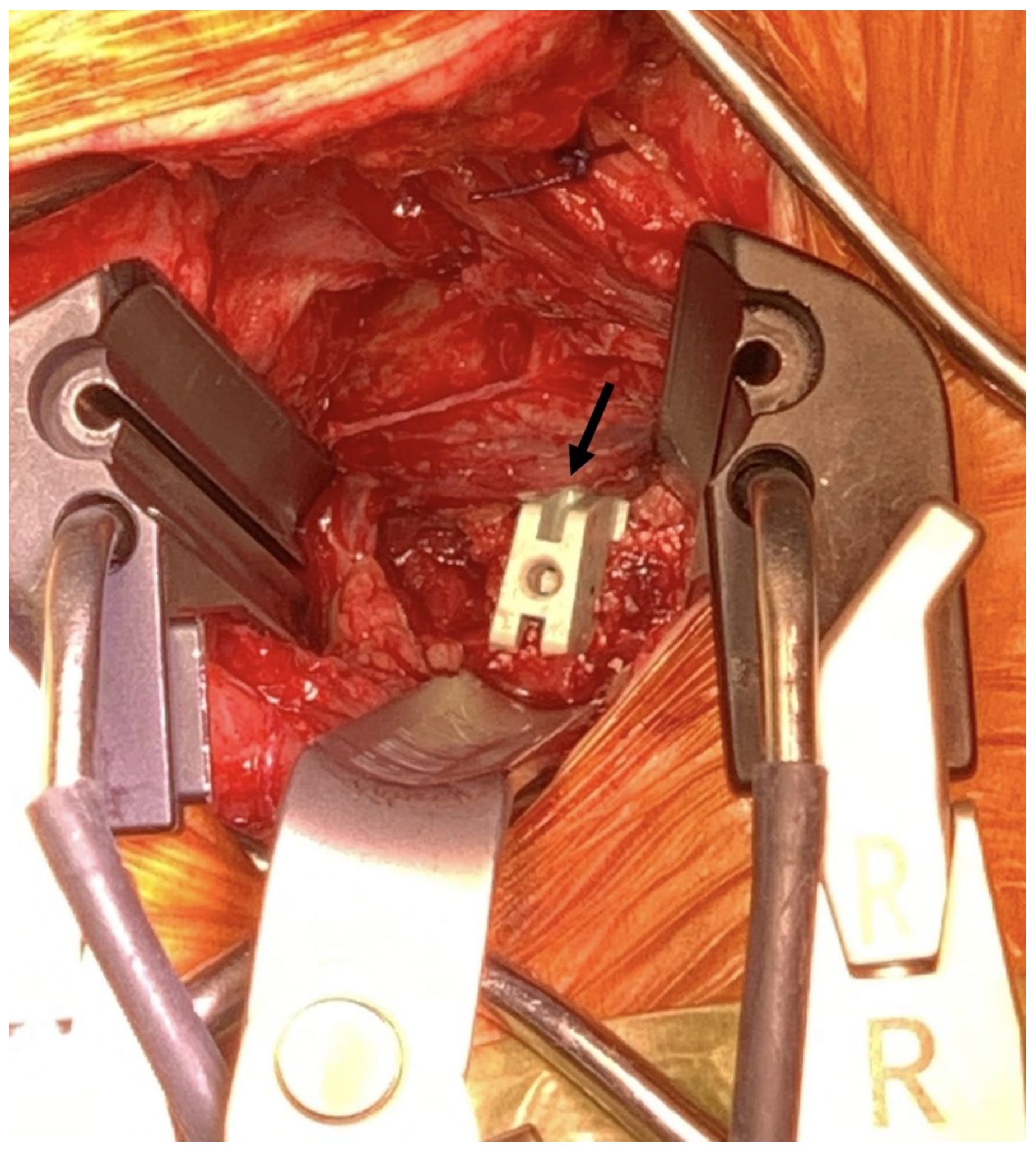

Figure 2

The implant cage was inserted under sufficient exposure. Arrow $(\rightarrow)$, the implant cage (Medtronic CLYDESDALE spine system) 


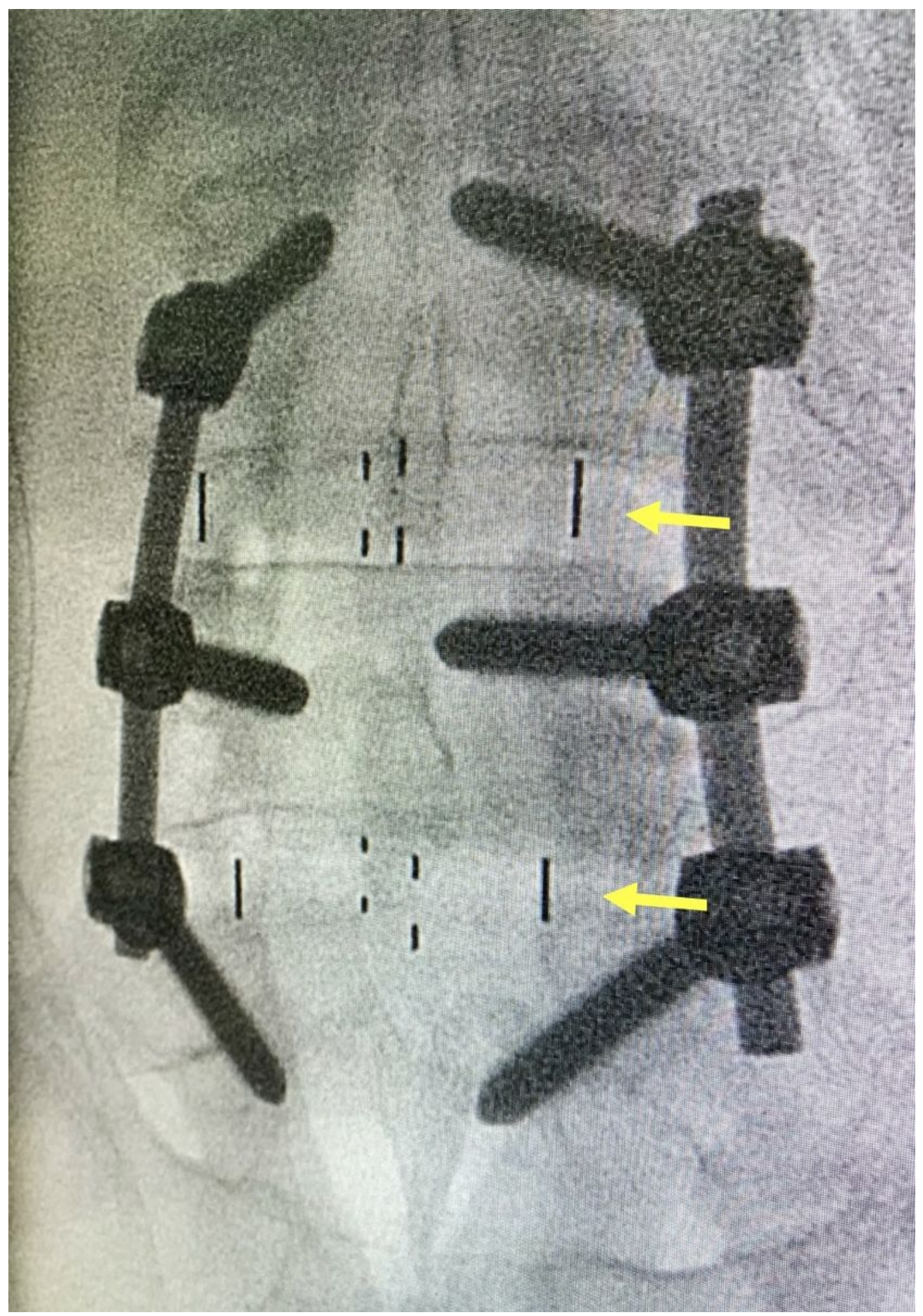

Figure 3

Position of the implant was confirmed by x-ray fluoroscopy after insertion. Arrow $(\rightarrow)$, implant cages in position 
$\mathrm{PI}-\mathrm{LL}=\frac{(\text { Age }-55)}{2}+3$

$\mathrm{PT}=\frac{(\text { Age }-55)}{3}+20$

$\mathrm{SVA}=2 \times($ Age -55$)+25$

Figure 4

Age-adjusted formulas for PI-LL, PT and SVA 

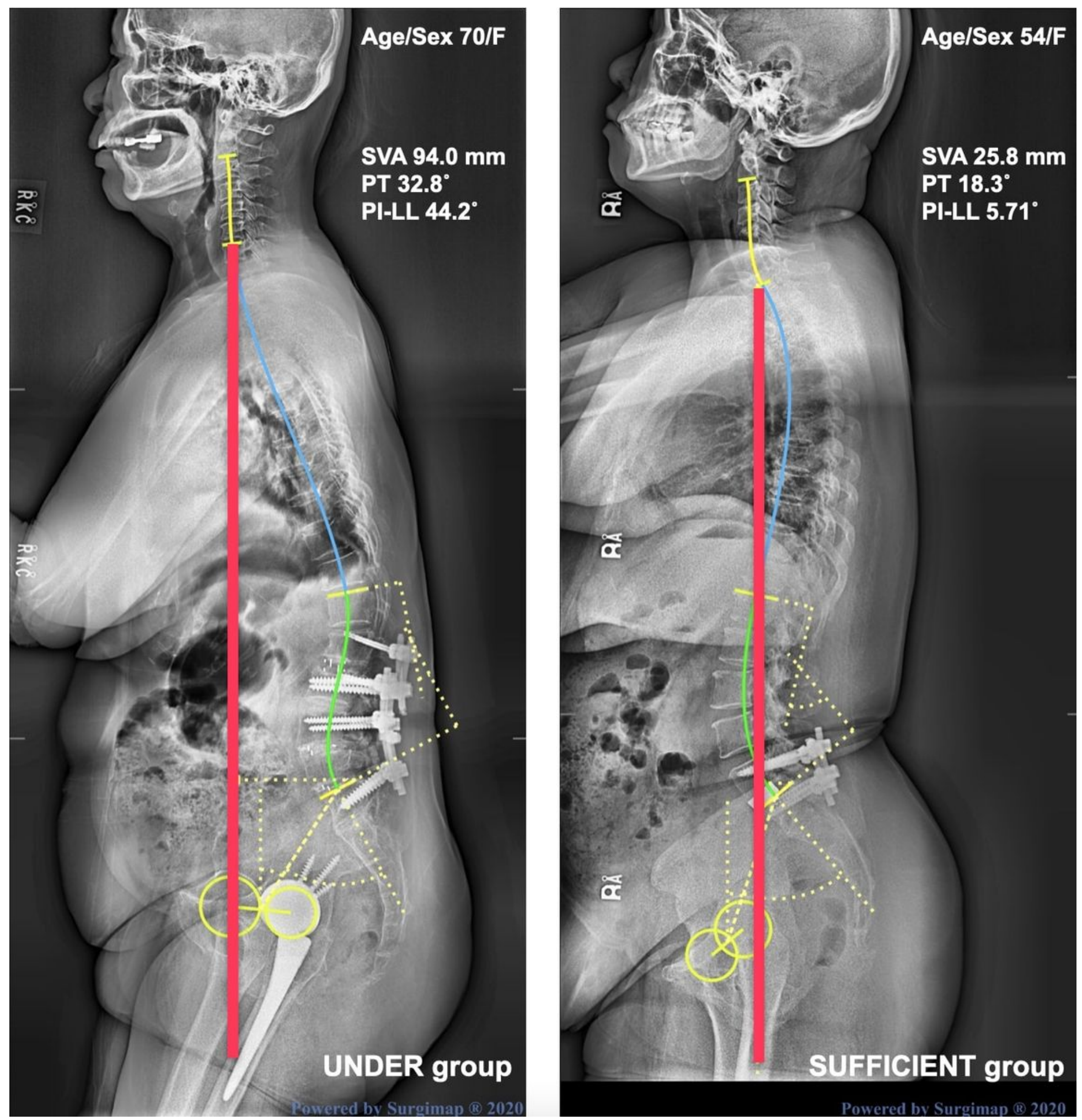

\section{Figure 5}

Demonstration for patients categorized as the "Under" or "Sufficient" group. Measurements were conducted using validated Surgimap surgical planning software (Nemaris Inc., New York, NY, United States) [20] 